\title{
Object-guided Spatial Attention in Touch: Holding the Same Object with Both Hands Delays Attentional Selection
}

\author{
Helge Gillmeister ${ }^{1}$, Julia Adler ${ }^{2}$, and Bettina Forster ${ }^{1}$
}

\begin{abstract}
Previous research has shown that attention to a specific location on a uniform visual object spreads throughout the entire object. Here we demonstrate that, similar to the visual system, spatial attention in touch can be object guided. We measured event-related brain potentials to tactile stimuli arising from objects held by observers' hands, when the hands were placed either near each other or far apart, holding two separate objects, or when they were far apart but holding a common object. Observers covertly oriented their attention to the left, to the right, or to both hands, following bilaterally presented tactile cues indicating likely tactile target location(s). Attentional modulations for tactile stimuli at attended compared to unattended locations were present in the time range of early somatosensory compo-
\end{abstract}

\section{INTRODUCTION}

For vision, it is known that attentional orienting toward locations can be both space- and object-based. Egly, Driver, and Rafal (1994) showed that covert attention can be shifted more rapidly between spatially separate locations when these appear on the same perceptual object than when they appear on different objects (see also Marino \& Scholl, 2005; Baylis \& Driver, 1992). In other words, space-based attentional costs of responding to visual signals at invalidly cued locations compared to validly cued locations were larger when the target appeared on another object than when it appeared on the same object at an equivalent distance. Egly et al. suggested that there may be interactions between a space-based system that selectively activates specific locations and an objectbased segmentation system that links separate locations on the basis of grouping operations dependent on the current input (see also Humphreys \& Riddoch, 1993). It has been proposed by several other researchers that the functional mechanism of this object-based spatial selection is founded on a strengthening of the sensory representation of an entire object because attention spreads throughout the object's boundaries (Davis, Driver, Pavani, \& Shepherd, 2000; Weber, Kramer, \& Miller, 1997; Vecera \& Farah, 1994).

\footnotetext{
${ }^{1}$ City University, London, UK, ${ }^{2}$ University of Leipzig, Germany
}

nents only when the hands were far apart, but not when they were near. This was found to reflect enhanced somatosensory processing at attended locations rather than suppressed processing at unattended locations. Crucially, holding a common object with both hands delayed attentional selection, similar to when the hands were near. This shows that the proprioceptive distance effect on tactile attentional selection arises when distant event locations can be treated as separate and unconnected sources of tactile stimulation, but not when they form part of the same object. These findings suggest that, similar to visual attention, both space- and object-based attentional mechanisms can operate when we select between tactile events on our body surface.

Recently, Martinez, Ramanathan, Foxe, Javitt, and Hillyard (2007) provided electrophysiological evidence that spatial attention directed to one part of a real or illusory object spreads throughout the entire object. Observers were cued to attend to one of four corners of a square, which was either intact or fragmented into four uneven sections (Experiment 1), and either illusory (induced by Kanisza figures) or absent (modified Kanisza figures, Experiment 2). Visual-evoked potentials were recorded to brief offsets of either attended or unattended corners. Offsets at attended locations gave rise to enhanced P1 and N1 components of the visual-evoked potential compared to offsets at unattended locations. Importantly, the space-based attentional effects over N1 (140-180 msec poststimulus onset) were found to be modified by the type of object configuration: Attentional effects in this time range were larger when the square was fragmented or absent than when it was an intact perceptual object, whether real or illusory. In other words, these results concur with those of Egly et al. (1994) in demonstrating that the prioritization of processing at one visual event location over another is smaller when these locations can be perceptually grouped. Because grouping effects also occurred for illusory objects, this study confirms that attentional selection can be truly object-based rather than guided by simple stimulus features such as parallel lines, as may have occurred in previous studies (see Marino \& Scholl, 2005; Avrahami, 1999). 
To the best of our knowledge, no study has so far investigated whether a similar object-based spatial-selection mechanism operates in touch. It is possible that the spatial selection between tactile events may be modulated by the processing of nonspatial stimulus attributes, such as object-related information. Several recent studies have demonstrated that, similar to visual and auditory systems, the somatosensory system extracts information about the identity and the spatial location of tactile stimuli in parallel, functionally specialized pathways (so-called what and where pathways; De Santis, Spierer, Clarke, \& Murray, 2007; Reed, Klatzky, \& Halgren, 2005; Van Boven, Ingeholm, Beauchamp, Bikle, \& Ungerleider, 2005; Forster \& Eimer, 2004), which may interact with one another in spatial selective attention.

To investigate whether the spread of spatial attention in touch can, like in vision, be modulated by object-based information, the present study used the so-called proprioceptive distance effect. Studies of this effect show that attentional selection between tactile events at different locations on the body is affected by their separation in external space, as perceived by proprioceptive feedback ("proprioceptive distance") (Schicke \& Röder, in press; Eimer, Forster, Fieger, \& Harbich, 2004; Soto-Faraco, Ronald, \& Spence, 2004; Lakatos \& Shepard, 1997; Driver \& Grossenbacher, 1996; Moscovitch \& Behrmann, 1994; Rinker \& Craig, 1994), analogous to effects of eccentricity on visual spatial attention (e.g., Intriligator \& Cavanagh, 2001; Driver \& Baylis, 1991; Eriksen \& Eriksen, 1974), and related to proprioceptive modulations of auditory attention (Simon-Dack \& Teder-Sälejärvi, 2008). For example, Driver and Grossenbacher (1996) demonstrated that response times to targets on an attended hand were slower when simultaneously presented distractors on the unattended hand were incongruent compared to when they were congruent with the target stimulation. Critically, this interference effect was less pronounced when the hands were far apart than when they were close together. ERP studies have confirmed that proprioceptive distance affects early somatosensory processing. Eimer et al. (2004) cued observers to direct attention to the left or to the right hand for a tactile discrimination task and found that tactile stimuli at attended compared to unattended locations resulted in modulations of both the somatosensory N140 component and the subsequent negative difference (Nd) at longer latencies (200-300 $\mathrm{msec})$. In the time range of the N140 component, effects of attention were more pronounced when the hands were far apart than when they were close together. These findings show that tactile spatial attention to the relevant hand operates more effectively when the distance of a distracting stimulus is increased in external space, although the somatotopic location of the distractor (irrelevant hand) remained unchanged, suggesting that tactile spatial selectivity operates in a spatial frame of reference that is based primarily on external (proprioceptive) rather than somatotopic coordinates (for a recent discussion on the deployment of both somatotopic and external reference frames in touch, see Azañón \& SotoFaraco, 2008).

In this present study, we compared the temporal dynamics of attentional selection for proprioceptively distant tactile events that arise from the same object (a bar held jointly by both hands) to events that arise from unconnected objects, which were either separated by the same distance or placed near each other. Tactile stimulators were embedded in two horizontal wooden bars, which observers held with their hands. The bars and the observers' hands were positioned either near each other (near condition) or far apart (far condition), or they were positioned far apart but solidly linked to each other via a connecting bar that could be attached between them (object condition). Tactile stimuli were preceded by tactile directional cues indicating the to-be-attended hand or directionally neutral cues instructing observers to attend to both hands. To assess the effects of covert spatial attention on somatosensory processing, we analyzed somatosensory ERPs in response to tactile stimuli when preceded by valid (indicating the hand that receives the tactile stimulus), invalid (indicating the opposite hand), and neutral (indicating both hands as possible stimulus locations) cues. Directionally neutral cues were included to investigate whether tactile attentional selection and its modulation by proprioceptive distance and object conditions primarily reflect enhancement of processing at attended locations or suppression of processing at unattended locations. Similar to other studies of visual (e.g., Luck et al., 1994), auditory (Schröger \& Eimer, 1997), and tactile (Forster \& Eimer, 2005) attention, attentional enhancement was defined as a difference between ERPs to validly and neutrally cued stimuli in the same direction as that of the general attentional effect (ERPs to validly vs. invalidly cued stimuli), and attentional suppression was defined as a difference between ERPs to invalidly and neutrally cued stimuli in the opposite direction as that of the general attentional effect.

As our aim was to investigate space- and object-based tactile attentional selection in the absence of any visuospatial information, we covered the hands and the bars from view. To manipulate observers' assumption of tactile events arising from common or separate objects, we included trials during which observers were cued to lift the bars off the tabletop a few times throughout each block. The lifting of the two bars when not connected (near and far conditions) would provide sensory feedback of two separate unimanual actions, whereas the lifting of the bars when connected to form a solid object (object condition) would result in analogous sensory feedback from both hands, which was expected to reinforce the assumption that tactile event locations were unconnected, or arose from a common object, respectively.

We expected to find an effect of proprioceptive distance on tactile attentional selection similar to Eimer et al. (2004). That is, effects of tactile attention (ERPs to validly vs. invalidly cued stimuli) were expected to be larger, or 
arise earlier, in the far condition than in the near condition, based on the assumption that the gain control over the flow of information exerted by spatial attention (e.g., Mangun \& Hillyard, 1995) is greater when the selected location (in our case, the hand) is more spatially separated from a distractor location (the other hand). We further hypothesized that, if tactile spatial attention is object-guided like visual spatial attention (Martinez et al., 2007; Egly et al., 1994), attention should spread throughout the object. That is, effects of attentional selection between the hands should be smaller, or arise later, when the hands are touching a common object (object condition) than when they are separated by an equivalent distance but are touching two separate objects (far condition). If, unlike in vision, attentional selection in touch is not object-guided and proprioceptive distance alone determines the extent to which processing at one tactile event location can be prioritized over that at another, there should be no difference between far and object conditions.

\section{METHODS}

\section{Participants}

Sixteen paid volunteers participated in the experiment. Four participants were excluded due to poor eye fixation control (see below) so that 12 participants (8 males, aged 20-37 years, $M=26.5$ years) remained in the sample. All participants were right-handed and had normal or corrected-to-normal vision by self-report.

\section{Stimuli and Apparatus}

Participants sat in a dimly lit experimental chamber, fixating on a small green LED about $65 \mathrm{~cm}$ in front of the body midline. A tabletop microphone was placed in front of them to record vocal response latencies. A video camera monitored participants throughout the experimental session. Participants were holding on to bars with their left and right hands and with their left and right index and middle fingers placed onto tactile stimulators that were embedded in the bars. The bars were constructed so that they were about $6 \mathrm{~cm}$ above the tabletop, affording a grip-like hand position (see Figure 1). In different conditions, the bars and therefore the hands were either placed close together (near condition), far apart (far condition), or far apart but solidly connected to one another through an additional bar between them (object condition). ${ }^{1}$ In the near condition, the bars were placed so that the left and the right index fingers were $6 \mathrm{~cm}$ apart. In the far and in the object conditions, left and right index fingers were $56 \mathrm{~cm}$ apart. The bars were held at a distance of about 30 to $45 \mathrm{~cm}$ from the body, depending on what distance felt comfortable for each participant while maintaining their grip. The bars and the participants' hands were covered from view by a black wooden board, which was placed about $30 \mathrm{~cm}$ above the tabletop.

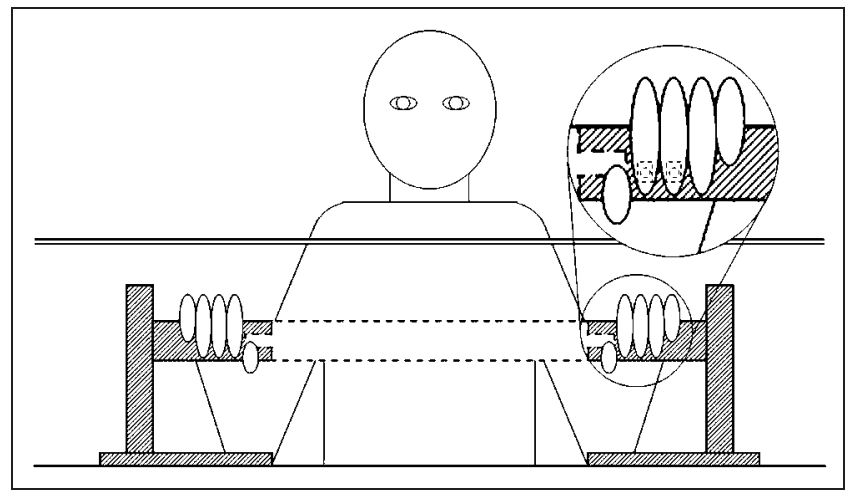

Figure 1. Experimental setup showing the bars participants held with their left and right hands in the far condition. Dotted lines indicate the outline of the connecting bar used in the object condition. The inset shows the location of the tactile stimulators used to present tactile cues (middle fingers), targets, and nontargets (index fingers).

Tactile stimuli were presented using four 12-V solenoids, driving a metal rod with a blunt conical tip to the fingertips of the left and right index and middle fingers, making contact with the finger whenever a current was passed through the solenoid. White noise $(65 \mathrm{~dB}$ sound pressure level [SPL], measured from the position of the participants' head) was continuously present to mask any sounds made by the tactile stimulators.

Tactile attentional cues were presented to the left and to the right middle finger simultaneously and consisted of simple taps or vibrations. Vibrations, generated by presenting a sequence of rapidly delivered brief pulses, were used as directional cues, indicating the left or the right hand as the likely target location. One of the two target locations was associated with a "flutter" vibration and the other with a "continuous" vibration. For "flutter" vibrations, the SOA between successive 6-msec pulses was $54 \mathrm{msec}$, corresponding to a rectangular stimulation frequency of about $16.7 \mathrm{~Hz}$. For "continuous" vibrations, the SOA between successive 2-msec pulses was $18 \mathrm{msec}$, corresponding to a frequency of $50 \mathrm{~Hz}$. Simple taps, where the rod of the solenoid contacted both middle fingers continuously for $300 \mathrm{msec}$, were used as directionally neutral cues (indicating both target locations). As the duration of each tactile cue (measured as the interval between the onset of the first pulse and the offset of the last pulse) would be different for the different types of cue, a 2-msec pulse was presented at $300 \mathrm{msec}$ following cue onset, rendering the cue duration $302 \mathrm{msec}$ for simple taps, "flutter" vibrations, and "continuous" vibrations alike. There was a fourth type of cue, indicating that participants should briefly lift up the bars, which consisted of a "flutter" vibration at all four fingers. Left and right middle fingers were contacted for $30 \mathrm{msec}$, followed by a 30-msec contact of both left and right index fingers, repeated five times, such that the total duration of the lift bar(s) cue was $300 \mathrm{msec}$. Tactile target and nontarget stimuli, which were presented unilaterally to the left or to the right index finger, consisted of single and double taps. 
For single taps (nontargets), the rod of the solenoid contacted the finger continuously for $200 \mathrm{msec}$. For double taps (targets), continuous contact was made for two periods of $85 \mathrm{msec}$, separated by a 30-msec pause, resulting in a total stimulus duration of $200 \mathrm{msec}$.

\section{Procedure}

The experiment consisted of 12 blocks, each consisting of 144 trials (for trial types and their frequency, see Table 1). Four blocks of each condition (near, far, and object) were presented successively, with the order counterbalanced across participants. Each trial started with a 302-msec presentation of the directional or nondirectional tactile cue or a 300-msec presentation of the lift bar(s) cue. We presented a tactile target or a nontarget stimulus to the left or to the right index finger 1000 msec after cue offset. Lift bar(s) cues were never followed by a tactile stimulus but by a 2000-msec pause before the onset of the next cue. Otherwise, the intertrial interval was 1000 msec. Participants were instructed to keep their eyes open and to fixate their gaze straight ahead on the green LED, to respond vocally ("pa") whenever a target stimulus (a double tap) was detected at the attended location, and to ignore all tactile nontarget stimuli (single taps) as well as target stimuli at the unattended location. Tactile cues indicated the most likely location for a target to occur. For six participants, a "flutter" vibration was associated with the left hand and a "continuous" vibration was associated with the right hand, and for the other six participants, cues and target locations were associated in the reverse manner. Neutral cues (simple taps) were associated with both target locations for all participants.

Table 1. Trial Types Defined by Conditions of Cueing and Target/Nontarget Presentation for One Block of 144 Trials

\begin{tabular}{lc}
\hline Cue Type & Target/Nontarget \\
(Number per Block) & (Number per Block)
\end{tabular}

Directional

Left (47) Left (12), right (3)/left (16), right (16)

Right (47) Left (3), right (12)/left (16), right (16)

\section{Nondirectional (Neutral)}

Left and right

$(44-48)^{\mathrm{a}}$

Left (6-8), ${ }^{\mathrm{a}}$ right $(6-8)^{\mathrm{a}} /$ left (16), right (16)

Lift $\operatorname{Bar}(s)(2-6)^{\mathrm{a}}$

Left and right refer to left hand and right hand, respectively.

${ }^{a}$ In all blocks, a total of 138 directional and neutral cueing trials were presented. An additional six trials were drawn from a pool of lift bar(s) and neutral cue trials, such that at least two, but never more than six, lift bar(s) trials were selected per block to vary the number of such trials across blocks. The remainder ( $0-4$ trials) were trials in which neutral cues were followed by left $(0-2)$ or right $(0-2)$ targets.

\section{EEG Recording}

EEG was recorded with $\mathrm{Ag}-\mathrm{AgCl}$ electrodes and on-line linked-earlobe reference from Fp1, Fp2, F3, Fz, F4, FC5, FC1, FCz, FC2, FC6, T7, C3, Cz, C4, T8, CP5, CP1, CP2, CP6, P7, P3, Pz, P4, P8, O1, and O2 (subset of the international 10-10 system). Horizontal EOG (HEOG) was recorded bipolarly from the outer canthi of both eyes. To encourage participants to lift the bars quickly and to enable monitoring their performance during lift bar(s) trials, we placed additional electrodes on the deltoid muscles of the left and right arms, although muscle EMG was recorded from the right arm only. Electrode impedance was kept below $2 \mathrm{k} \Omega$ for reference and ground electrodes and below $5 \mathrm{k} \Omega$ for all other electrodes, and the impedances of the earlobe electrodes were kept as equal as possible. A BrainAmps amplifier and a BrainVision Recorder (version 1.02) and Analyzer (version 1.05) software (Brain Products GmbH, Munich, Germany) were used for recording and off-line analysis of the EEG data. Amplifier band-pass was 0.01-100 Hz, and digitization rate was $500 \mathrm{~Hz}$. EEG was filtered off-line with a digital lowpass filter of $40 \mathrm{~Hz}$. EEG and HEOG were epoched in separate off-line analysis and were extracted for a period from $100 \mathrm{msec}$ before to $400 \mathrm{msec}$ after the onset of the tactile stimulus. To check for eye movements in the interval between cue and tactile stimulus onsets, we also extracted epochs for the 1000-msec period between the onset of the cue and the onset of the tactile stimuli. Averaged HEOG waveforms obtained in this interval were scored for systematic deviations of eye position, indicating a tendency to move the eyes toward the cued side. Four participants were disqualified due to residual HEOG deflections exceeding $\pm 4 \mu \mathrm{V}$ in the cue-tactile stimulus interval. Analyses were only conducted for ERPs obtained in response to tactile nontarget stimuli. Trials with horizontal eye movements (HEOG exceeding $\pm 40 \mu \mathrm{V}$ relative to baseline), eye blinks or other artifacts (a voltage exceeding $\pm 70 \mu \mathrm{V}$ at any electrode relative to baseline) measured in the interval starting $100 \mathrm{msec}$ before cue onset and ending $400 \mathrm{msec}$ after the onset of the nontarget stimulus, were excluded from analysis.

\section{ERP Analysis}

ERPs to tactile nontargets were averaged relative to a 100-msec prestimulus baseline for all combinations of cue type (valid vs. invalid vs. neutral), stimulated hand (left vs. right), and hand condition (near vs. far vs. object). ERP mean amplitudes were computed within successive measurement windows centered on the latencies of early somatosensory ERP components P100 and N140 (96-150 msec poststimulus). To investigate longer-latency effects of attention, we also computed mean amplitudes between 170 and 300 msec poststimulus. For each of these two time windows, statistical analyses of ERP mean amplitudes were conducted initially to compare the effect of hand condition (near vs. far 
vs. object) on overall effects of attention (validly vs. invalidly cued trials). ${ }^{2}$ These analyses were conducted separately for lateral recording sites F3/4, F7/8, FC1/2, FC5/6, C3/4, CP1/2, $\mathrm{CP} 5 / 6, \mathrm{P} 3 / 4, \mathrm{P} 7 / 8$, and $\mathrm{O} 1 / 2$ and for midline electrodes Fz, $\mathrm{FCz}, \mathrm{Cz}$, and Pz. Lateral recording sites were grouped into four quadrants of five electrodes each, as defined by lateral and anteroposterior axes (anterior-ipsilateral: F3/4i, F7/8i, FC1/2i, FC5/6i, and C3/4i; anterior-contralateral: F3/4c, F7/ 8c, FC1/2c, FC5/6c, and C3/4c; posterior-ipsilateral: CP1/2i, $\mathrm{CP} 5 / 6 \mathrm{i}, \mathrm{P} 3 / 4 \mathrm{i}, \mathrm{P} 7 / 8 \mathrm{i}$, and $\mathrm{O} 1 / 2 \mathrm{i}$; posterior-contralateral: $\mathrm{CP} 1 /$ 2c, $\mathrm{CP} 5 / 6 \mathrm{c}, \mathrm{P} 3 / 4 \mathrm{c}, \mathrm{P} 7 / 8 \mathrm{c}$, and O1/2c). For lateral recording sites, repeated measures ANOVAs were conducted for the within-subject factors cue type (valid vs. invalid), hand condition (near vs. far vs. object), hemisphere (contralateral vs. ipsilateral electrode sites), anterior-posterior location (anterior vs. posterior electrode sites), and electrode (see above). For recording sites along the midline, repeated measures ANOVAs were conducted for the within-subject factors cue type (valid vs. invalid), hand condition (near vs. far vs. object), and electrode (Fz vs. FCz vs. Cz vs. Pz). Follow-up analyses were conducted for each combination of the three hand conditions (near vs. far, far vs. object, and near vs. object) in each time window and for the subsets of electrodes where overall effects of hand condition on attention were found. In these analyses, further follow-up analyses were conducted to identify effects of attention in each of the three hand conditions separately, for each time window, and for the subsets of electrodes where effects of hand condition on attention were found. To test whether attentional effects were based on enhancement or suppression of processing at attended or unattended locations, respectively, we conducted separate analyses comparing conditions of cue type (valid vs. neutral and neutral vs. invalid) for each hand condition, time window, and subsets of electrodes where overall effects of attention were found. When appropriate, Greenhouse-Geisser adjustments to the degrees of freedom were applied.

\section{RESULTS}

\section{Behavioral Performance}

Participants responded vocally upon detection of infrequent targets at attended locations on validly and neutrally cued trials. Response times were analyzed with repeated measures ANOVAs for the factors cue type (valid vs. neutral) and hand condition (near vs. far vs. object). Responses to targets were faster in validly $(562 \mathrm{msec})$ than that in neutrally cued trials $(602 \mathrm{msec})$, Cue Type, $F(1$, $11)=60.4, p \leq .001$, irrespective of hand condition, Hand Condition $\times$ Cue Type, $F(2,22)=1.5, p=.251$. Because participants were asked not to respond to targets in invalidly cued trials to maximize attentional effects in the ERPs, behavioral effects of attention (i.e., RTs to targets at attended vs. unattended locations) could not be measured, which precludes the relation of behavioral to ERP effects in this study.

\section{Somatosensory ERPs}

Figure 2 shows somatosensory ERPs in response to tactile nontarget stimuli at a subset of electrodes ipsilateral and contralateral to the stimulated hand as well as at midline electrode Cz. ERPs are presented separately for stimuli at validly (black lines), invalidly (gray lines), and neutrally cued locations (dashed lines), when the hands were positioned near (Figure 2A), far (Figure 2B), and far but connected through an object (Figure 2C). Figure 3 shows ERPs to stimuli at validly and invalidly cued locations for one representative electrode over somatosensory cortex contralateral to the stimulated hand $(\mathrm{C} 3 / 4 \mathrm{c}$, panel A) and voltage difference maps (activations in response to validly cued vs. invalidly cued stimuli) for the time range in which attentional effects differed between hand conditions (panel B). ERPs and voltage difference maps are shown for each of the three hand conditions (near: top panel; far: middle panel; object: bottom panel).

In the near condition (Figures 2A and 3, top panel), modulations of attention were only present at later processing stages, in the time range following the N140 component, where they appeared as an $\mathrm{Nd}$ between tactile stimuli at validly and invalidly cued locations. In the far condition (Figures 2B and 3, middle panel), attentional modulations were present much earlier. ERPs in response to tactile stimuli at validly cued locations showed an enhanced positivity compared with stimulation at invalidly cued locations, which started in the time range of the P100 component and extended into the time range of the N140 component. These attentional modulations appeared to be far more prominent over anterior than over posterior electrode sites. Similar to the near condition, there was also an $\mathrm{Nd}$ effect of attention. In contrast to the early somatosensory modulations present in the far condition, when the hands were far apart but connected by an object (Figures 2C and 3, bottom panel), effects of attention appeared to be only present as an $\mathrm{Nd}$ effect but not at earlier stages.

These informal observations were tested with an overall ANOVA investigating effects of hand condition (near vs. far vs. object) on attention (valid vs. invalid) in the early and in the late time window.

\section{Effects of Hand Condition on Early Attentional Selection}

For the time window of the earlier components (96$150 \mathrm{msec}$ ), effects of attention differed as a function of hand condition, specifically over anterior electrodes for lateral electrode sites (Hand Condition $\times$ Cue Type $\times$ Anterior-Posterior Location, $F(1,11)=3.8, p=.040$; Hand Condition $\times$ Cue Type $\times$ Anterior-Posterior Location $\times$ Electrode, $F(1,11)=3.3, p=.016)$ as well as for midline electrode sites (Hand Condition $\times$ Cue Type $\times$ Electrode, $F(1,11)=4.6, p=.013)$. For lateral sites, there were no effects of hemisphere (Hand Condition $\times$ Cue 
Figure 2. Grand-averaged somatosensory ERPs elicited at a subset of sites ipsilateral (i) and contralateral (c) to the stimulated hand and at midline electrode $\mathrm{Cz}$ by tactile nontarget stimuli at validly cued locations (black lines), at invalidly cued locations (gray lines), and at neutrally cued locations (dashed lines) in the 400-msec interval following stimulus onset. (A) Near condition. (B) Far condition. (C) Object condition.

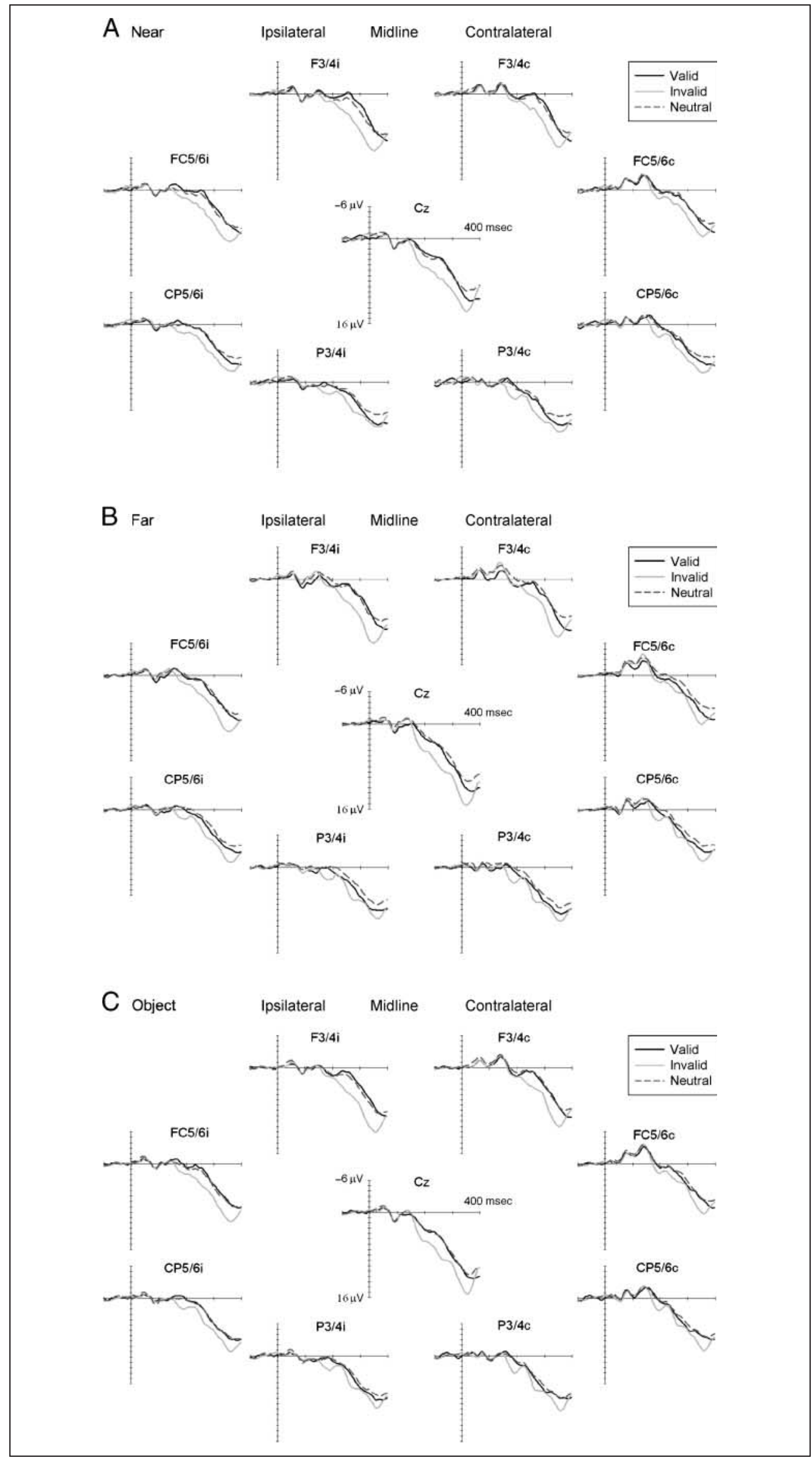


Figure 3. (A) Enlarged image of ERPs elicited at one representative electrode (C3/4c) by tactile stimuli at validly cued (black lines) and invalidly cued (gray lines) locations. The shaded area indicates the 96- to $150-\mathrm{msec}$ time range. (B) Voltage difference maps (activations elicited by validly cued vs. invalidly cued stimuli) for the 96- to 150-msec time range in which attentional effects differed between hand conditions. Black contour lines indicate levels of negative difference; white contour lines indicate levels of positive difference. Black $\mathrm{x}$ indicates the location of C3/4c. Top: near condition. Middle: far condition. Bottom: object condition.

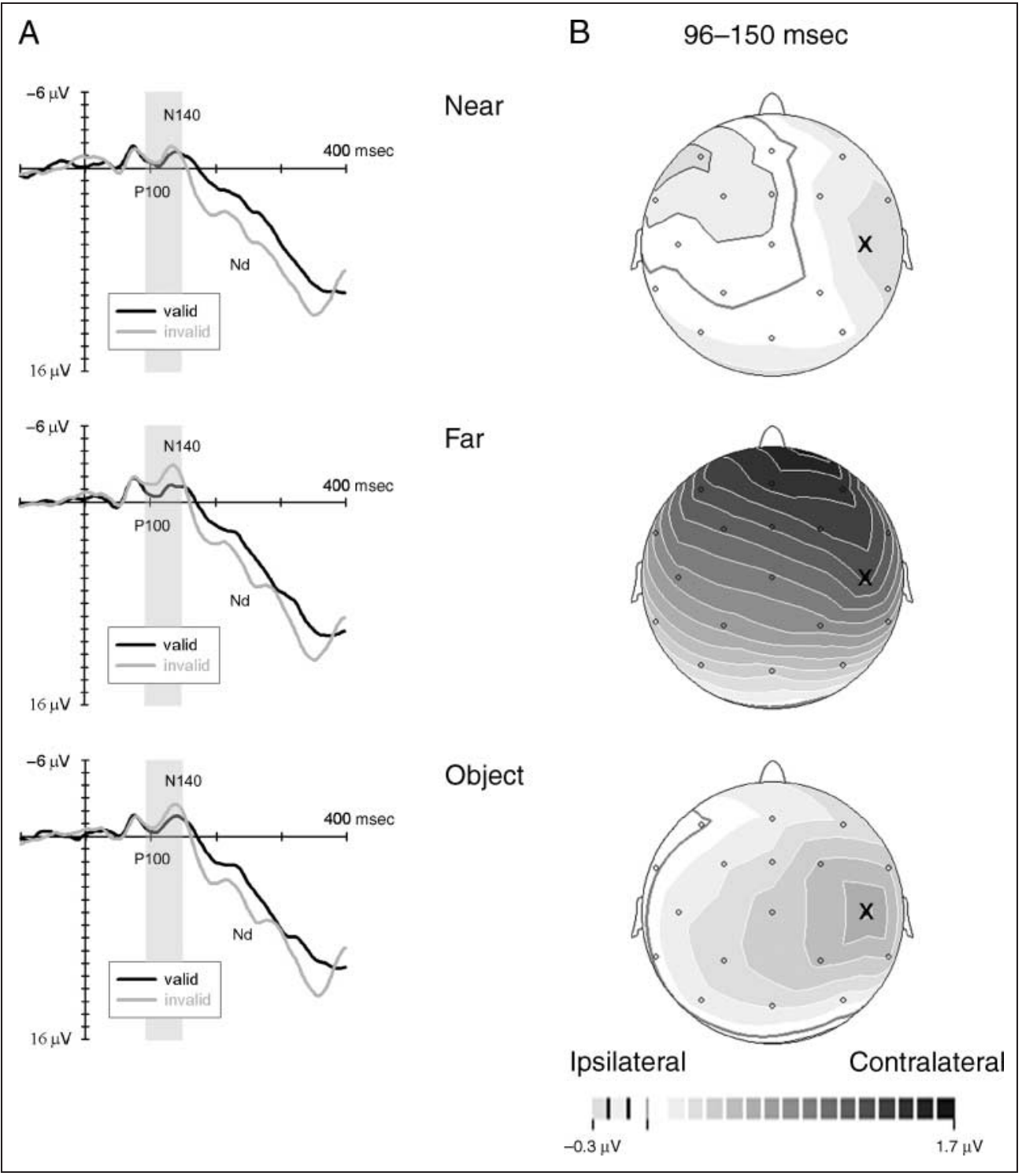

Type $\times$ Hemisphere, $F(1,11)<1, p=.975$; Hand Condition $\times$ Cue Type $\times$ Anterior-Posterior Location $\times$ Hemisphere, $F(1,11)<1, p=.618)$. Simple effects analyses revealed that there were significant effects of attention in the far condition over lateral anterior $(p=.007)$ but not over posterior sites $(p=.317)$ and not over either lateral anterior or posterior sites in both near or object conditions $(p \geq .394)$. Effects of attention in the far condition were present for all lateral anterior electrodes $(p \leq .029)$ as well as for anterior midline electrodes $\mathrm{Fz}, \mathrm{FCz}$, and $\mathrm{Cz}(p \leq$ $.038)$ and marginal or absent for lateral posterior electrodes $(p \geq .075)$ as well as for posterior midline electrode $\mathrm{Pz}$ $(p=.431)$. In both near and object conditions, effects of attention were absent for all lateral $(p \geq .190)$ and midline $(p \geq .318)$ electrodes.

Therefore, we separately compared the effects of hand condition on attentional selection for lateral anterior electrode sites and anterior midline sites Fz, FCz, and $\mathrm{Cz}$ for each combination of the three hand conditions (near vs. far, far vs. object, and near vs. object). Effects of attention were larger in the far compared with the near condition (Hand Condition $\times$ Cue Type, $F(1,11) \geq 5.7, p \geq .036$, for analyses of lateral and midline sites), irrespective of hemisphere $($ Hand Condition $\times$ Cue Type $\times$ Hemisphere, $F(1,11)<1, p=.687$, for lateral sites). Effects of attention were marginally larger in the far compared with the object condition for lateral electrode sites (Hand Condition $\times$ Cue Type, $F(1,11)=4.1, p=.067)$, irrespective of hemisphere (Hand Condition $\times$ Cue Type $\times$ Hemisphere, $F(1$, $11)<1, p=.669)$. For midline sites, effects of attention were larger in the far compared with the object condition as a function of electrode (Hand Condition $\times$ Cue Type $\times$ Electrode, $F(1,11)=5.7, p=.029)$. Separate analyses for each electrode showed that effects of attention were larger in the far compared with the object condition for electrode $\mathrm{Fz}$ (Hand Condition $\times$ Cue Type, $F(1,11)=8.1, p=.016$ ) but not for electrode $\mathrm{FCz}$ or $\mathrm{Cz}$ (Hand Condition $\times$ Cue Type, $F(1,11)<2.6, p \geq .136)$. Importantly, effects of 
attention did not differ between near and object conditions (Hand Condition $\times$ Cue Type, $F(1,11)<1, p \geq .591$, for analyses of lateral and midline sites), irrespective of hemisphere (Hand Condition $\times$ Cue Type $\times$ Hemisphere, $F(1$, 11) $<1, p=.952$, for lateral sites).

For the same set electrodes, analyses of attentional effects were also conducted for each of the three hand conditions separately. For the far condition, effects of attention (Cue Type, $F(1,11) \geq 10.9, p \leq .007$, for analyses of lateral and midline sites) differed as a function of electrode for lateral sites (Cue Type $\times$ Electrode, $F(1,11)=$ $3.9, p=.036$ ), irrespective of hemisphere (Cue Type $\times$ Hemisphere $\times$ Electrode, $F(1,11)=1.6, p=.212$ ), and differed marginally as a function of electrode for midline sites (Cue Type $\times$ Electrode, $F(1,11)=4.4, p=.053$ ). Separate analyses for each electrode showed that effects of attention were present for all lateral electrodes (Cue Type, $F(1,11) \geq 6.3, p \leq .029)$ as well as for all midline electrodes (Cue Type, $F(1,11) \geq 5.6, p \leq .038)$, but they were largest over frontal electrodes F3/4 and Fz (Cue Type, $F(1,11) \geq$ $14.4, p \leq .003$ ) followed by frontocentral electrodes FC1/2 and FCz (Cue Type, $F(1,11) \geq 10.5, p \leq .008$ ) and were smallest for central electrodes C3/4 and Cz (Cue Type, $F(1,11) \geq 14.4, p \leq .038)$ and the more temporally situated frontal and frontocentral electrodes F7/8 and FC5/6 (Cue Type, $F(1,11) \geq 14.4, p \leq .029)$.

For the near condition, there were no effects of attention (Cue Type, $F(1,11)<1, p \geq .831$, for analyses of lateral and midline sites), independently of electrode (Cue Type $\times$ Electrode site, $F(1,11)<1, p \geq .663$, for analyses of lateral and midline sites). For lateral sites, there was a marginally significant interaction between attention and hemisphere (Cue Type $\times$ Hemisphere, $F(1,11)=4.1, p=$ .067), but simple effects analyses indicated that there were no effects of attention at either ipsi- or contralateral sites $(p \geq .625)$.

For the object condition, there were also no effects of attention (Cue Type, $F(1,11)<1, p \geq .394$, for analyses of lateral and midline sites), independently of electrode (Cue Type $\times$ Electrode, $F(1,11) \leq 1.7, p \geq .214$, for analyses of lateral and midline sites) or hemisphere (Cue Type $\times$ Hemisphere, $F(1,11)=2.1, p=.179$, for lateral sites).

\section{Effects of Hand Condition on Late Attentional Selection}

For the time window of the Nd (170-300 msec), effects of attention (Cue Type, $F(1,11) \geq 23.3, p \leq .001$, for analyses of lateral and midline sites) did not differ as a function of hand condition (Hand Condition $\times$ Cue Type, $F(1$, 11) $<1, p \geq .540$, for analyses of lateral and midline sites). Attentional effects were larger for ipsilateral than for contralateral electrode sites (Cue Type $\times$ Hemisphere, $F(1$, $11)=7.9, p=.017$; Cue Type $\times$ Hemisphere $\times$ Electrode, $F(1,11)=6.8, p=.009)$, especially over anterior electrode sites (Cue Type $\times$ Hemisphere $\times$ Anterior-Posterior Location, $F(1,11)=38.7, p<.001$; Cue Type $\times$ Hemisphere $\times$
Anterior-Posterior Location $\times$ Electrode, $F(1,11)=13.5$, $p<.001)$. Attentional effects also differed as a function of electrode for midline sites (Cue Type $\times$ Electrode, $F(1$, $11)=5.9, p=.024)$. Simple effects analyses indicated that, across all hand conditions, attentional effects were marginal for anterior contralateral electrode F7/8c ( $p=.051)$ but otherwise present for all electrodes in all lateral quadrants $(p \leq .008)$ as well as for all midline electrodes $(p \leq .005)$, but they were largest over frontal electrodes $\mathrm{F} 3 / 4 \mathrm{i}$ and $\mathrm{Fz}$, frontocentral electrodes FC1/2i, FC1/2c, and FCz, central electrodes $\mathrm{C} 3 / 4 \mathrm{i}$ and $\mathrm{Cz}$, and centroparietal electrodes $\mathrm{CP} 1 / 2 \mathrm{i}$ and $\mathrm{CP} 1 / 2 \mathrm{c}(p \leq .005)$.

\section{Effects of Attentional Enhancement and Suppression}

In this study, we also included trials where attention was cued to both hands and compared somatosensory ERPs evoked by stimuli in these trials (neutrally cued stimuli) with those evoked in trials where only one hand was selectively attended to assess the relative contributions of attentional enhancement (validly vs. neutrally cued stimuli) and suppression (invalidly vs. neutrally cued stimuli) to overall attentional effects (validly vs. invalidly cued stimuli). Somatosensory ERPs in response to tactile nontarget stimuli at validly, invalidly, and neutrally cued locations are shown in Figure 2 separately for the three hand conditions. The earliest attentional modulations were evident for the time range of the P100 and N140 components in the far condition (Figure 2B). These early modulations appeared to reflect attentional enhancement rather than suppression; that is, ERPs in response to stimuli at validly cued locations differed not only from those to stimuli at invalidly cued locations but also from those at neutrally cued locations over most electrode sites, whereas ERPs to neutrally and invalidly cued stimuli did not differ. In addition to these early modulations, attentional modulations were also present as Nd effects in all three hand conditions. In contrast to earlier attentional modulations, Nd effects on somatosensory processing appeared to reflect primarily attentional suppression; that is, ERPs to stimuli at invalidly cued locations differed not only from those stimuli at validly cued locations but also from those at neutrally cued locations, whereas ERPs to validly and neutrally cued stimuli did not differ.

To formally test these observations, we carried out ANOVAs for anterior lateral and midline electrode sites for the time window of earlier attentional effects in the far condition and for all electrode sites for the time window of the Nd for all three hand conditions. The contribution of attentional enhancement to earlier attentional effects was shown by differences between waveforms to validly and neutrally cued stimuli (Cued Type, $F(1,11) \geq 8.5, p \leq$ .014 , for analyses of lateral and midline sites) in the absence of differences between waveforms to neutrally and invalidly cued stimuli (Cue Type, $F(1,11)<1, p \geq .703$, for analyses of lateral and midline sites). Similar to the overall attentional effect (validly vs. invalidly cued stimuli), atten- 
tional enhancement for validly compared with neutrally cued stimuli was present irrespective of hemisphere (Cue Type $\times$ Hemisphere, $F(1,11)=1.5, p=.243$, for lateral sites). Enhancement did not differ across electrodes (Cue Type $\times$ Electrode, $F(1,11) \leq 2.6, p \geq .128$, for analyses of lateral and midline sites).

For the $\mathrm{Nd}$, effects of attentional suppression were shown by differences between waveforms to neutrally and invalidly cued stimuli (Cue Type, $F(1,11) \geq 29.0, p<$ .001 , for analyses of lateral and midline sites) in the absence of differences between waveforms to validly and neutrally cued stimuli (Cue Type, $F(1,11) \leq 1.3, p \geq .273$, for analyses of lateral and midline sites). Similar to the overall attentional effect (validly vs. invalidly cued stimuli), attentional suppression for invalidly compared with neutrally cued stimuli was larger for ipsilateral than for contralateral electrode sites specifically over anterior electrode sites (Cue Type $\times$ Hemisphere $\times$ Anterior-Posterior Location, $F(1$, $11)=5.4, p=.040)$, although it was not larger for ipsilateral sites overall (Cue Type $\times$ Hemisphere, $F(1,11)=1.5, p=$ .248). Attentional suppression also differed as a function of electrode (Cue Type $\times$ Electrode, $F(1,11) \geq 4.9, p \leq$ .031 , for analyses of lateral and midline sites). Although suppression was present for all lateral and midline electrodes $(p \leq .003)$, it was largest for frontocentral electrodes FC1/2i, FC1/2c, and FCz, central electrodes C3/4i, $\mathrm{C} 3 / 4 \mathrm{c}$, and $\mathrm{Cz}$, and centroparietal electrodes CP1/2i and $\mathrm{CP} 1 / 2 \mathrm{c}(p<.001)$.

\section{DISCUSSION}

To investigate whether tactile spatial attention can be object based, analogous to visual spatial attention (Martinez et al., 2007; Egly et al., 1994), we recorded ERP correlates of the proprioceptive distance effect on tactile spatial attention (Eimer et al., 2004; Soto-Faraco et al., 2004; Driver \& Grossenbacher, 1996) and tested whether spatial attentional selection between proprioceptively distant hands is attenuated, or arises later, when the hands are connected by a jointly held object. Based on the study of Eimer et al. (2004), which showed that ERP correlates of tactile spatial attention are modulated by the proprioceptive distance of the site of tactile stimulation (hands), we devised a task in which participants were holding either one or two objects while their hands were positioned either near or far apart. We compared attentional selection for tactile events on the hands in three different conditions: when tactile stimulation arose from two separate objects that observers held in their hands, when the hands were placed either near together or far apart, and when tactile stimulation arose from a common object held by both hands that were placed far apart. We induced the perception of whether tactile event locations were separate or connected by occasionally cueing observers to lift the object(s) they were holding as part of each experimental block. To investigate tactile attentional selection in the absence of any engagement of visuospatial orienting mechanisms, we cued observers' attention tactually rather than visually as done in most previous studies of tactile spatial attention. In addition, we included directionally neutral cues to explore the relative contributions of enhancement at attended locations and suppression at unattended locations to effects of tactile attentional selection.

Previous studies have shown that the representation of hand positions in external space profoundly affects tactile attentional selection (Soto-Faraco et al., 2004; Driver \& Grossenbacher, 1996). In line with equivalent effects on early somatosensory processing (Eimer et al., 2004), the present study shows that proprioceptive information about hand location modulates the mechanisms underlying tactile spatial attention. For this modulation to occur, integration between tactile and proprioceptive information must have taken place prior to the operation of attentional selection. We found that effects of attentional selection arose earlier (96-150 msec poststimulus) when the hands were placed far apart than when they were near each other (170-300 msec poststimulus). These earlier attentional effects were present over anterior electrodes as an enhanced positivity for attended compared to unattended stimuli. Interestingly, effects of attentional selection were also delayed (170-300 msec poststimulus) when hands were placed far apart but were holding the same object. In fact, evoked responses in the object condition did not differ from those observed when the hands were placed near each other. These findings show for the first time that object-based information about tactile events on the hands affects the temporal dynamics of tactile attentional selection, suggesting that tactile spatial attention, analogous to visual attention, can be both space- and object-based.

Our findings also show that early effects (96-150 msec poststimulus) of proprioceptive distance on attentional selection (far condition) consist primarily of an enhancement of processing for stimuli at the attended hand rather than of a suppression of processing at the unattended hand. At later processing stages, attentional selection was unaffected by the distance between the hands or whether they were holding an object; all three hand conditions showed a similar $\mathrm{Nd}$ between tactile stimuli at attended and unattended locations. Attentional effects in the time range of the $\mathrm{Nd}$ were found to consist primarily of a suppression of processing for tactile stimuli at invalidly cued locations compared with those at validly or neutrally cued locations, which is in line with previous findings (Forster \& Eimer, 2005). In addition, our results suggest that early enhancement of tactile processing at attended locations is only present when tactile stimuli originate from separate sources that are sufficiently distant in external space. These findings are in contrast to Driver and Grossenbacher's (1996) suggestion that the more effective attentional selection between proprioceptively distant hands is due to better suppression of events at the unattended, distractor hand rather than enhancement of events at the attended, target hand. However, in their study, participants were presented with tactile stimuli given simultaneously to both 
hands. It is conceivable that mechanisms underlying tactile spatial selection may differ with stimulus presentation and task demands. Similar to Forster and Eimer (2005), we have found that attentional enhancement, if present, arises at earlier stages than suppression, which is in contrast to similar studies of visual (Luck et al., 1994) and auditory attention (Schröger \& Eimer, 1997), where suppression typically precedes enhancement, and suggests that the component stages of attentional mechanisms in somatosensation differ from those in other modalities. In contrast to our results, however, Forster and Eimer found that attentional suppression accompanied enhancement at early stages of visually cued tactile selection. This difference between the two studies suggests that mechanisms of tactile spatial attention may also be affected by cueing modality.

The most important novel finding of this study is that the proprioceptive distance effect on early tactile attentional selection disappeared when the two hands were separated in external space but connected through a jointly held object. In this condition, effects of attentional selection did not arise until later stages of processing, similar to when the hands were placed near each other. The present findings mirror analogous findings made for visual spatial attention and show for the first time that tactile attention can be both space- and object-based. Behavioral (e.g., Egly et al., 1994) and electrophysiological (e.g., Martinez et al., 2007) studies have shown that visual spatial attention spreads along object boundaries: Effects of attentional selection between two equidistant locations are smaller when these locations form part of the same perceptual object than when they do not. Similarly, the present study demonstrates that effects of attentional selection between the hands are delayed when the hands are connected by a jointly held object than when they are separated by the same distance but hold two unconnected objects. This suggests that, at these stages, no location along a jointly held object receives prioritized processing because, like visual attention, tactile attention spreads along object boundaries. In addition to a spacebased selection system that activates specific tactile event locations over others, the tactile modality may have an object-based system that links or segments tactile event locations on the basis of grouping operations that can or cannot be performed on the current input. Previous studies have shown that nonspatial attributes of tactile stimuli (e.g., frequency) are selected in parallel with spatial attributes in functionally segregated pathways (De Santis et al., 2007; Reed et al., 2005; Van Boven et al., 2005; Forster \& Eimer, 2004). Our results extend these findings by showing that object-related information can modulate the spatial processing of tactile events, suggesting that specialized somatosensory what and where pathways may interact at early stages of spatial processing. Our findings also suggest that tactile and visual attentional systems may operate in similar ways. Just as the spread of spatial attention throughout an entire visual object may be useful for object perception and indeed occurs at the level of regions implicated in object encoding (see Martinez et al., 2007), the absence of prioritized processing for locations along an object held by the two hands might be useful for purposes such as the bimanual handling of objects.

In line with previous studies, we have shown that tactile attentional selection is modulated by the proprioceptive distance between stimulus locations (Eimer et al., 2004; Driver \& Grossenbacher, 1996). In addition, our findings suggest that larger or earlier effects of tactile attentional selection with greater separation between the hands are driven by the system's assumption that proprioceptively distant event locations can be represented as unconnected sources of information. The proprioceptive distance effect on tactile attentional selection necessitates that proprioceptive information about current limb position is integrated with tactile processing before the operations of tactile spatial attention mechanisms. If tactile attentional selection is object-based, prior knowledge about whether tactile event locations arise from the same or different objects must be integrated with tactile processing in a similar manner.

Recently, Helbig and Ernst (2007) showed that prior knowledge about two events arising from a common source can affect how these events are treated by the perceptual system. Observers judged the shape of objects they simultaneously touched and viewed either when they had direct vision of their hand (colocated visual and haptic information) or when vision of their hand was provided via a mirror (creating a spatial separation between visual and haptic information). Typically, with greater spatial separations between the sources of visual and haptic information, their beneficial effect on one another gradually declines (Gepshtein, Burge, Ernst, \& Banks, 2005), but integration did not differ between viewing conditions in Helbig and Ernst's study. This suggests that when observers have prior knowledge about object identity, that is, when they believe that vision and touch provide redundant information about the same object, information from both modalities is integrated, overcoming even substantial spatial separations between them. Helbig and Ernst argued that the system must first decide whether information from different sources (different sensory modalities) pertain to the same object or event (see also Ma \& Pouget, 2008). If so, perceptual integration, which does not typically occur for spatially separate multimodal events, can take place in the same way as it would for colocated events. In line with this, we suggest that prior knowledge modulated the integration of proprioceptive information with tactile input in our study. Because tactile event locations could be treated as arising from a common source of stimulation in our object condition, but as arising from separate sources in our far condition, the proprioceptive or spatial distance between them was reflected in attentional modulations of early somatosensory processing stages only in the far condition. 
Our findings suggest that when the hands were proprioceptively distant but connected by a common object, attentional selection operated as if the hands were near, at least at early stages of processing. The question that arises from these findings is whether object-based effects occur despite proprioceptive distance, such that distance information is essentially preserved, or whether the nature of the proprioceptive information is fundamentally changed through the assumption that tactile events on both hands have a common source. That is, object-based effects may lead to a representation of the hands as "near" or as "connected," thereby essentially changing the current body schema with respect to the functional relationship between the hands. Future studies should address the question of how the functional relationship between the hands is affected, for example, by investigating the consequences of object-guided selection for the preparation of hand movements.

\section{Acknowledgments}

The authors thank Keith Hodgson (www.antcreative.com) for the artwork on the cover of this issue. This research was supported by a grant from the Biotechnology and Biological Sciences Research Council (BBSRC).

Reprint requests should be sent to Helge Gillmeister, Department of Psychology, City University, 10 Northampton Square, London EC1V 0HB, UK, or via e-mail: helge.gillmeister.1@city.ac.uk.

\section{Notes}

1. To ensure that tactile stimulation at one end of the bar did not travel along the object and contaminate ERPs to stimuli at the other end, we recorded EEG during the presentation of stimuli at the side held by one hand, at the other side (not held), and when no stimuli were presented and found that no somatosensory ERPs were evoked from stimulation at the other side of the object, similar to when no stimuli were presented.

2. We also analyzed earlier components P45 (30-60 msec) and N80 (60-90 msec), but these analyses are not reported because there were no effects of or interactions with attention for lateral or midline electrodes, $F(1,11) \leq 2.2, p \geq .136$, except the following. Over $\mathrm{P} 45$, there was a very marginal interaction between attention, hand condition, and hemisphere, $F(1,11)=2.7$, $p=.095$, but pairwise comparisons showed no attentional effects at any levels of hand condition and hemisphere $(p \geq$ .208). Over N80, there was an interaction between attention, hemisphere, and anterior-posterior location, $F(1,11)=7.4$, $p=.020$, but pairwise comparisons showed no attentional effects at any levels of hemisphere and anterior-posterior location $(p \geq .125)$.

\section{REFERENCES}

Avrahami, J. (1999). Objects of attention, objects of perception. Perception \& Psychophysics, 61, 1604-1612.

Azañón, E., \& Soto-Faraco, S. (2008). Changing references frames during the encoding of tactile events. Current Biology, 18, 1044-1049.

Baylis, G. C., \& Driver, J. (1992). Visual parsing and response competition: The effect of grouping factors. Perception $\varepsilon$ Psychophysics, 51, 145-162.

Davis, G., Driver, J., Pavani, F., \& Shepherd, A. (2000). Reappraising the apparent costs of attending to two separate visual objects. Vision Research, 40, 1323-1332.

De Santis, L., Spierer, L., Clarke, S., \& Murray, M. M. (2007). Getting in touch: Segregated somatosensory what and where pathways in humans revealed by electrical neuroimaging. Neuroimage, 37, 890-903.

Driver, J., \& Baylis, G. C. (1991). Target-distractor separation and feature integration in visual attention to letters. Acta Psychologica (Amsterdam), 76, 101-119.

Driver, J., \& Grossenbacher, P. (1996). Multimodal constraints on tactile spatial attention. In T. Innui \& J. McClelland (Eds.), Attention and performance (Vol. XVI, pp. 209-235). Cambridge, MA: MIT Press.

Egly, R., Driver, J., \& Rafal, R. D. (1994). Shifting visual attention between objects and locations: Evidence from normal and parietal lesion subjects. Journal of Experimental Psychology: General, 123, 161-177.

Eimer, M., Forster, B., Fieger, A., \& Harbich, S. (2004). Effects of hand posture on preparatory control processes and sensory modulations in tactile-spatial attention. Clinical Neurophysiology, 115, 596-608.

Eriksen, B. A., \& Eriksen, C. W. (1974). Effects of noise letters upon the identification of a target letter in a nonsearch task. Perception \& Psychophysics, 16, 143-149.

Forster, B., \& Eimer, M. (2004). The attentional selection of spatial and nonspatial attributes in touch: ERP evidence for parallel and independent processes. Biological Psychology, 66, 1-20.

Forster, B., \& Eimer, M. (2005). Covert attention in touch: Behavioral and ERP evidence for costs and benefits. Psychophysiology, 42, 171-179.

Gepshtein, S., Burge, J., Ernst, M. O., \& Banks, M. S. (2005). The combination of vision and touch depends on spatial proximity. Journal of Vision, 5, 1013-1023.

Helbig, H. B., \& Ernst, M. O. (2007). Knowledge about a common source can promote visual-haptic integration. Perception, 36, 1523-1533.

Humphreys, G. W., \& Riddoch, M. J. (1993). Interaction between space-based and object-based systems revealed through neuropsychology. In D. E. Meyer \& S. Kornblum (Eds.), Attention and performance (Vol. XIV). Hillsdale, NJ: Erlbaum.

Intriligator, J., \& Cavanagh, P. (2001). The spatial resolution of visual attention. Cognitive Psychology, 43, 171-216.

Lakatos, S., \& Shepard, R. N. (1997). Time-distance relations in shifting attention between locations on one's body. Perception \& Psychophysics, 59, 557-566.

Luck, S. J., Hillyard, S. A., Mouloua, M., Woldorff, M. G., Clark, V. P., \& Hawkins, H. L. (1994). Effects of spatial cuing on luminance detectability: Psychophysical and electrophysiological evidence for early selection. Journal of Experimental Psychology: Human Perception and Performance, 20, 887-904.

Ma, W. J., \& Pouget, A. (2008). Linking neurons to behavior in multisensory perception: A computational review. Brain Research, 1242, 4-12.

Mangun, G. R., \& Hillyard, S. A. (1995). Attention: Mechanisms and models. In M. D. Rugg \& M. G. H. Coles (Eds.), Electrophysiology of mind: Event-related potentials and cognition (pp. 40-85). Oxford: Oxford University Press.

Marino, A. C., \& Scholl, B. J. (2005). The role of closure in defining the "objects" of object-based attention. Perception \& Psychophysics, 67, 1140-1149.

Martinez, A., Ramanathan, D. S., Foxe, J. J., Javitt, D. C., \& Hillyard, S. A. (2007). The role of spatial attention in the 
selection of real and illusory objects. Journal of Neuroscience, 27, 7963-7973.

Moscovitch, M., \& Behrmann, M. (1994). Coding of spatial information in the somatosensory system: Evidence from patients with neglect following parietal lobe damage. Journal of Cognitive Neuroscience, 6, 151-155.

Reed, C. L., Klatzky, R. L., \& Halgren, E. (2005). What vs. where in touch: An fMRI study. Neuroimage, 25, 718-726.

Rinker, M. A., \& Craig, J. C. (1994). The effect of spatial orientation on the perception of moving tactile stimuli. Perception \& Psychophysics, 56, 356-362.

Schicke, T., \& Röder, B. (in press). Common anatomical and external coding for hands and feet in tactile attention: Evidence from event-related potentials. Journal of Cognitive Neuroscience.

Schröger, E., \& Eimer, M. (1997). Costs and benefits in event-related potential signs of auditory spatial attention. Quarterly Journal of Experimental Psychology, 50, 457-474
Simon-Dack, S. L., \& Teder-Sälejärvi, W. A. (2008). Proprioceptive cues modulate further processing of spatially congruent auditory information. A high-density EEG study. Brain Research, 1220, 171-178.

Soto-Faraco, S., Ronald, A., \& Spence, C. (2004). Tactile selective attention and body posture: Assessing the multisensory contributions of vision and proprioception. Perception \& Psychophysics, 66, 1077-1094.

Van Boven, R. W., Ingeholm, J. E., Beauchamp, M. S., Bikle, P. C., \& Ungerleider, L. G. (2005). Tactile form and location processing in the human brain. Proceedings of the National Academy of Sciences, U.S.A., 102, 12601-12605.

Vecera, S. P., \& Farah, M. J. (1994). Does visual attention select objects or locations? Journal of Experimental Psychology: General, 123, 146-160.

Weber, T. A., Kramer, A. F., \& Miller, G. A. (1997). Selective processing of superimposed objects: An electrophysiological analysis of object-based attentional selection. Biological Psychology, 45, 159-182. 